\title{
Application of multifunctional nanomaterials in cancer vaccines (Review)
}

\author{
BIAO FU ${ }^{1 *}$, XIAOMEI HUANG ${ }^{1 *}$, JIAQI DENG $^{2}$, DAIJIAO GU $^{1}$, QIBING MEI $^{3}$, \\ MINGMING DENG ${ }^{1}$, SHIXIAO TANG ${ }^{1}$ and MUHAN LÜ ${ }^{1}$
}

\author{
${ }^{1}$ Department of Gastroenterology, The Affiliated Hospital of Southwest Medical University, Luzhou, Sichuan 646000; \\ ${ }^{2}$ School of Foreign Languages, Southwest Medical University, Luzhou, Sichuan 646000; ${ }^{3}$ Laboratory of Molecular Pharmacology, \\ Department of Pharmacology, School of Pharmacy, Southwest Medical University, Luzhou, Sichuan 646000, P.R. China
}

Received August 6, 2017; Accepted December 15, 2017

DOI: 10.3892/or.2018.6206

\begin{abstract}
Tumor immunotherapy has been in development for more than a century. With the rapid developments in biotechnology research in recent years, immunotherapy has become a promising oncotherapy strategy after surgery, chemotherapy and radiotherapy. Cancer vaccines are a promising new treatment strategy and the application of nanotechnology in cancer vaccines, greatly enhances their effectiveness. Such applications indicate the bright prospects of tumor immunotherapy. The multifunctional nanomaterials used in cancer vaccines and their practical application in specific cancer vaccines are hereby reviewed. In addition, a preliminary analysis of the current and prospective use of nanotechnology with the purpose of providing solutions to cancer vaccine challenges is presented.
\end{abstract}

\section{Contents}

1. Introduction

2. Multifunctional nanomaterials

3. Application of nanotechnology in cancer vaccines

4. Conclusions and future perspectives

\section{Introduction}

Traditional cancer treatments such as surgery, radiation therapy and drug treatments, present certain limitations. As a

Correspondence to: Professors Shixiao Tang or Muhan Lü, Department of Gastroenterology, The Affiliated Hospital of Southwest Medical University, Zhongshan campus, no. 319, section 3, Zhongshan Road, Luzhou, Sichuan 646000, P.R. China

E-mail: 26111377@qq.com

E-mail: 1vmuhan990@163.com

*Contributed equally

Key words: multifunctional nanomaterial, cancer vaccine, cancer immunotherapy, cross-presentation, tumor microenvironment new treatment strategy, biological immunotherapy has become the fourth leading tumor-therapy approach. At present, the commonly used immunotherapy methods include adoptive immunotherapy ( $\mathrm{T}$ and NK cells), cytokine, gene and antitumor antibody therapy, immunological checkpoint (PD-1/PDL1, CTLA-4) blockade therapy and cancer vaccines (1). Cancer vaccines, by expressing specific and immunogenic tumor antigens (polypeptides, DNA and RNA), activate or enhance the antitumor immunity of the body with the aid of adjuvants such as cytokines and chemokines to kill and remove tumor cells (2). Cancer vaccines can be divided into the following categories, according to the different types of antigens: tumor cell, dendritic cell (DC), peptide and nucleic acid vaccines (3). Unlike prophylactic vaccines, commonly used in healthy individuals, therapeutic cancer vaccines administered to cancer patients are designed to eliminate cancer cells by enhancing the immune response of the patient (4). This therapeutic vaccination mobilizes a variety of immune mechanisms to specifically attack and destroy cancer cells. Thus, compared with traditional treatments, cancer vaccines, in principle, may be used to inhibit further growth of advanced cancers and/or recurrent tumors.

The application of nanotechnology has become popular in cancer vaccine research in recent years (5). Due to their biosafety capacity for site-specific delivery of antigens and enhancement of the bioavailability of antigens, nanomaterials are widely used as cancer vaccine-carriers or adjuvants (6). Nanomaterials can deliver targeted antigens and adjuvants (7), prevent their rapid degradation (8) and increase the retention time of tumor antigens in lymphatic and tumor tissues (9), thereby increasing the efficacy and safety of vaccines. Cancer vaccines produce immune effects that firstly require antigen-presenting cells (APCs), especially DCs, to effectively ingest and present tumor-associated antigens (TAAs) (10). Soluble proteins are generally not easily adsorbed by APCs, but antigen-loaded nanoparticles are comparable in size to pathogens and are more easily recognized and ingested by APCs, thereby improving the immunogenicity of vaccines (11). Currently, some nanomaterials have demonstrated good carrier or adjuvant activity in animal studies (12). In the present review, we present the functions and applications of nanomaterials in cancer 


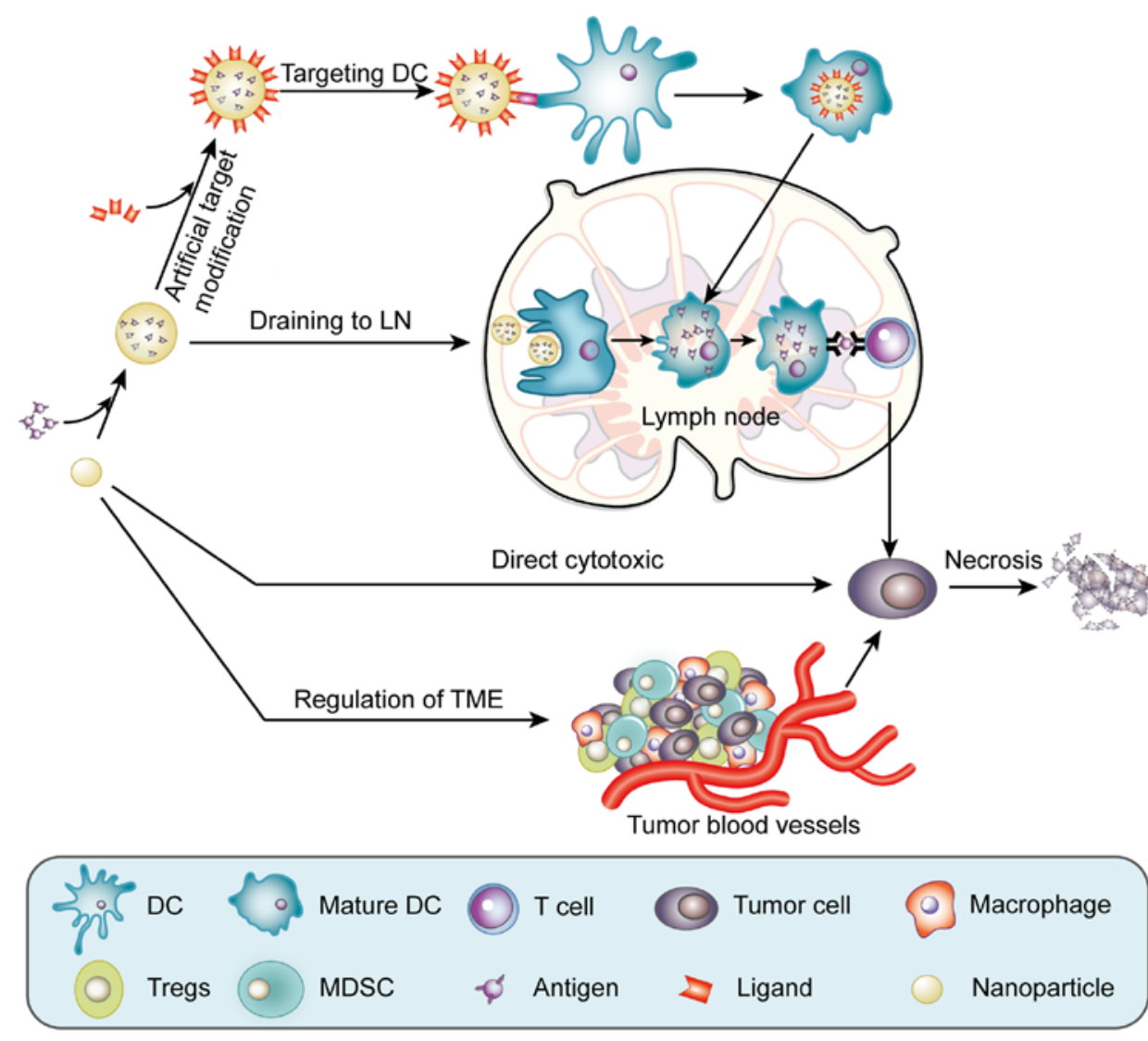

Figure 1. Functions of multifunctional nanomaterials in cancer vaccines. Nanoparticles can encapsulate tumor antigens to protect them from degradation. After the surface of a nanoparticle is modified with the relevant targeting ligand, a nanovaccine can target DCs. After peripheral immature DC intake of nanovaccines, the DC matures and migrates to the lymph nodes. Some nanoparticles can enhance the drainage of the vaccine to the lymph nodes, which increases the concentration of nanovaccines in the lymph nodes and is conducive to DC uptake of the vaccine. In the lymph nodes, the nanovaccine is presented to T cells, producing antigen-specific cellular immunity. Furthemore, some nanomaterials can directly kill tumor cells. In addition, some nanoparticles can inhibit the development of tumor cells by regulating the TME. DC, dendritic cell; LN, lymph node; Tregs, regulatory T cells; MDSC, myeloid-derived suppressor cell.

vaccines. The various functions of nanomaterials in cancer vaccines are depicted in Fig. 1. The advantages and disadvantages of different cancer vaccines and the corresponding types and functions of the nanoparticles in the vaccines are summarized in Table I.

\section{Multifunctional nanomaterials}

Protect and delay antigen release. Relative to conventional cancer vaccines, some nanomaterials can protect antigens from enzyme-mediated degradation and are able to delay the release of antigens, which is conducive to the continued role of the antigen (8).

Song et al (13) combined a PEG-b-PLACL aqueous solution with $\operatorname{Span}^{\circledR} 85$ and squalene to form a stable isotropic PELC emulsion. Subsequently, the pre-emulsified material was dispersed in RAH polypeptide antigen solution to form uniform nanoparticles. Experiments revealed that the PELC formulation could delay the release of antigens in vitro and in vivo. PELC formulation protected the antigen from rapid degradation and enhanced the effectiveness of the vaccine. Furthermore, the vaccine effectively increased the number of $\mathrm{CD}^{+}{ }^{+} \mathrm{T}$ cells and $\mathrm{RAH}$-specific $\mathrm{CD}^{+} \mathrm{T}$ cells, promoted the secretion of IFN- $\gamma$, enhanced the cytotoxic T-cell response and antitumor effect and inhibited tumor growth in mice.
Co-delivery of the antigen and the adjuvant. In cancer vaccine immunotherapy, simultaneous delivery of the antigen and the adjuvant can improve the efficiency of cancer vaccines (14). Nanomaterials deliver antigens into the body, activate the immune system and induce cellular and humoral immune responses. The main functions of the adjuvant are to accelerate a lasting immune response, reduce the dosage of the antigen and activate a specific cellular immune response.

Standley et al (15) synthesized nanoparticles with acrylamide as a monomer, that can degrade in the acidic lysosomal environment and covalently bind $\mathrm{CpG}$ and encapsulate ovalbumin (OVA) to co-deliver antigen and $\mathrm{CpG}$. Compared with free OVA, OVA-NPs or OVA + CpG, DCs can significantly increase the expression of IL-12 and costimulatory molecules, resulting in stronger antigen-specific T-cell immune responses. Fox et al (16) used liposomes to carry the TLR4-agonist glucopyranoside A and the TLR7-agonist imiquimod. The Th1 phenotype of $\mathrm{CD}^{+} \mathrm{T}$ cells increased significantly compared with the use of a single agonist. This finding indicates that nanoparticles that co-deliver multiple immunostimulants possess a synergistic effect.

Efficient draining to lymph nodes. Lymph nodes located in lymphatic drainage pathways play an important role in the humoral immune response and immune cell activity (17). 
Table I. Summary of multifunctional nanomaterials for cancer vaccines.

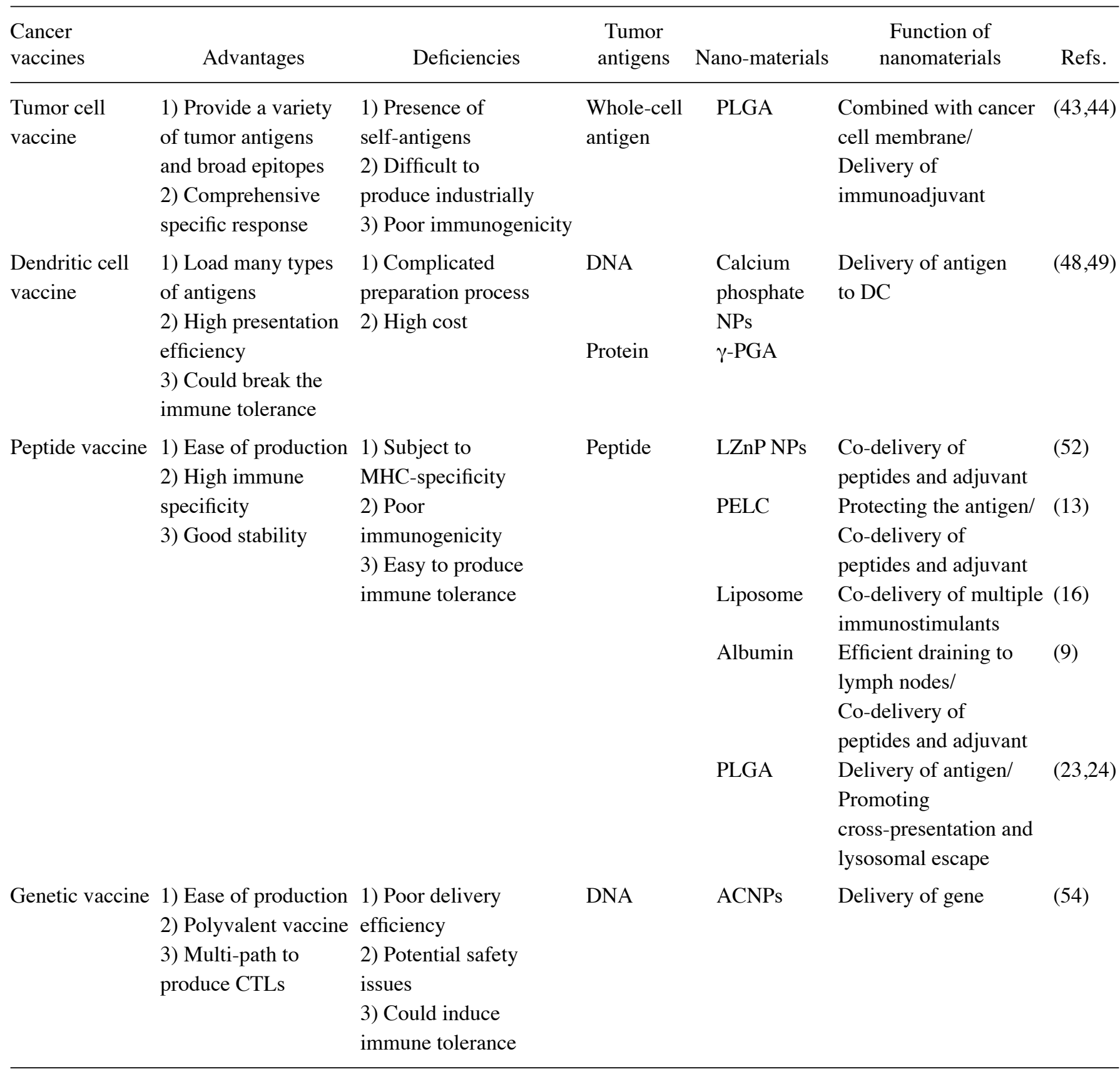

Nanocarriers can effectively drain the vaccine to the lymphoid tissue, increase the concentration of the vaccine in the lymph nodes, prolong the residence time of the vaccine in lymphoid tissue and control the release of the vaccine and the adjuvant. This function facilitates the antigen presentation and induces T-cell activation to enhance the effects of cancer vaccines.

Liu et al (9) used albumin as a carrier to target a cancer vaccine to the lymph nodes. In vivo, serum albumin is abundant and can transport fatty acids from the blood into lymphatic vessels and lymph nodes. Using this function of albumin, it was conjugated with a lipid, a peptide antigen and a $\mathrm{CpG}$ to form an amphiphilic molecular cancer vaccine (AMPH vaccine) that was inoculated into tumor-bearing mice. After inoculation of the structurally optimized CpG-DNA/AMPH vaccine in mice, it significantly accumulated in the lymph nodes, thereby enhancing the antitumor effect of T cells, while significantly reducing systemic toxicity.

Targeting DCs. APCs are of critical importance in transporting antigens from peripheral circulation to local organized lymphoid tissue. DCs are the most powerful APCs and are at the heart of initiation, regulation and maintenance of immune responses. They can present antigens through MHC class I and MHC class II molecular pathways, provide sufficient co-stimulatory signals and play a key role in initiation and regulation of the T-cell antitumor immune response. DCs express many surface receptors, such as mannose, DEC-205, CD40, CD11c and DC-SIGN receptors (18). The surface of nanoparticles encapsulating an antigen can bind to targeting ligands (mannose, anti-CD11c and anti-DEC205) 


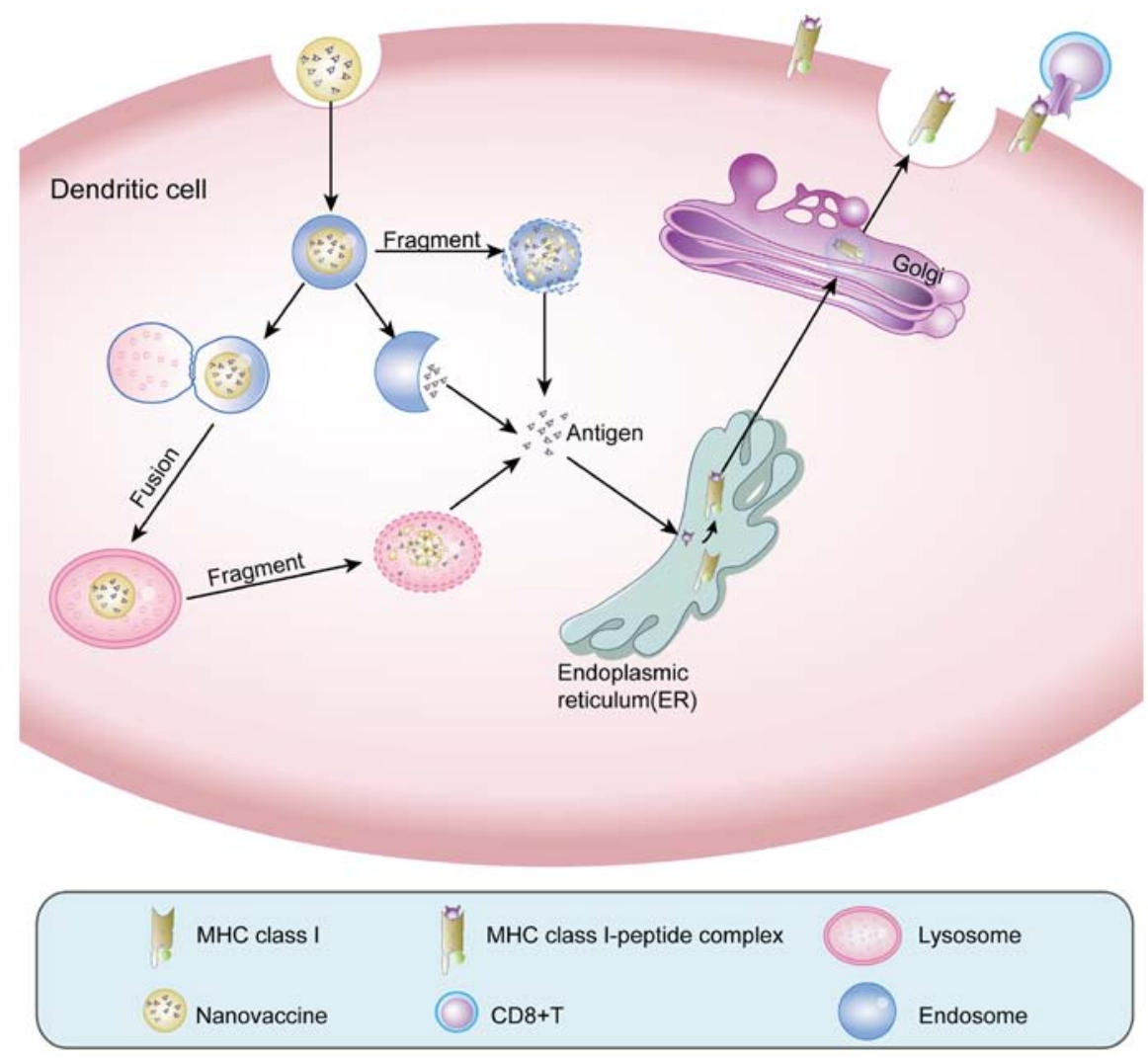

Figure 2. Lysosomal escape and cross-presentation. Nanovaccines are phagocytosed by DCs into endosomes. On one hand, certain nanomaterials can destroy the endosomal membrane or fuse with the endosome to release the antigen into the cytoplasm. On the other hand, the endosome fuses with the lysosomal membrane, delivering the nanovaccine into the lysosome. In the acidic environment of the lysosome, nanomaterials turn the lysosome into a proton sponge, destroying the lysosome and releasing the antigen into the cytoplasm. Through these effects, the exogenous antigens in the nanovaccine are converted into endogenous antigens. Thus, the antigen can enter the endoplasmic reticulum and bind to MHC I molecules, which are delivered to the Golgi apparatus for further modification and are finally expressed on the cell surface and recognized by $\mathrm{CD} 8^{+} \mathrm{T}$ cells and thus, activate $\mathrm{CD} 8^{+} \mathrm{T}$ cells. $\mathrm{DCs}$, dendritic cells.

to specifically deliver the antigen to DCs, thereby promoting binding and endocytosis of the targeting ligands to enhance the effect of the vaccine $(19,20)$.

Guo et al (20) developed erythrocyte membrane-enveloped PLGA nanoparticles containing the antigenic peptide (hgp10025-33) and the TLR4 agonist monophosphoryl lipid A (MPLA). The nanoparticle surface was mannose-loaded, allowing the nanoparticles to be actively targeted by lymphoid organ DCs and promote accumulation in the lymph nodes. The vaccine was inoculated into melanoma-bearing mice and prolonged the antitumor time effect, inhibited tumor growth and metastasis and effectively increased IFN- $\gamma$ secretion and $\mathrm{CD} 8^{+}$ T cell response.

Promoting cross-presentation and lysosomal escape. Antigens in cancer vaccines are exogenous antigens. In theory, the antigens activate $\mathrm{CD} 4^{+} \mathrm{T}$ cells through an MHC II presentation pathway in vivo. However, the antitumor effect is mainly produced by activation of the $\mathrm{CD} 8^{+} \mathrm{T}$ cell-mediated immunity and $\mathrm{CD} 4^{+} \mathrm{T}$ cells play a supporting role (21). Some nanovaccines can release antigens into the cytoplasm after being taken up by DCs. Subsequently the antigen escapes degradation by lysosomes and thus, can be presented by MHC class I molecules through the endogenous pathway and the $\mathrm{CD} 8^{+} \mathrm{T}$ cells can be activated. Exogenous antigens are presented via MHC I molecules instead of MHC II molecules, which is called crosspresentation (22) (Fig. 2). After entering the cell, the antigen is not damaged by the acidic lysosomal environment and the large number of enzymes and may produce biological effects to activate $\mathrm{CD} 8^{+}$cytotoxic $\mathrm{T}$ cells through cross-presentation to kill cancer cells. Therefore, it is important to assist the antigen escape from lysosomes in order to improve the vaccine effect. Nanocarriers are often designed based on this premise.

Shen et al (23) demonstrated that PLGA nanoparticles can enhance exogenous antigen escape from the endosome to the cytoplasm, leading to presentation of the antigen in conjunction with MHC I molecules through cross-presentation. Saluja et al (24) encapsulated the MART-127-35 peptide (melanoma-associated antigen) with PLGA nanoparticles and modified the nanoparticle surface with DEC-205 ligands to obtain a nanovaccine capable of targeting DCs. The results indicated that PLGA nanoparticles promoted the antigen uptake by DCs and facilitated the lysosomal escape, thus promoting cross-presentation of the exogenous antigens as well as activation and proliferation of $\mathrm{CD} 8^{+} \mathrm{T}$ cells.

Yuba et al (25) synthesized highly $\mathrm{pH}$-sensitive liposomes that were stable at neutral $\mathrm{pH}$ and sensitive to slight changes in $\mathrm{pH}$ in a weakly acidic environment, which rendered them unstable. The nanomaterials were combined with OVA to treat tumor-bearing mice. The nanovaccine could effectively be taken up by DCs, where it then fused with the endosomal membrane in the acidic environment and was released into the cytoplasm, leading to cross-presentation, which effectively induced an antigen-specific cellular immune response. 


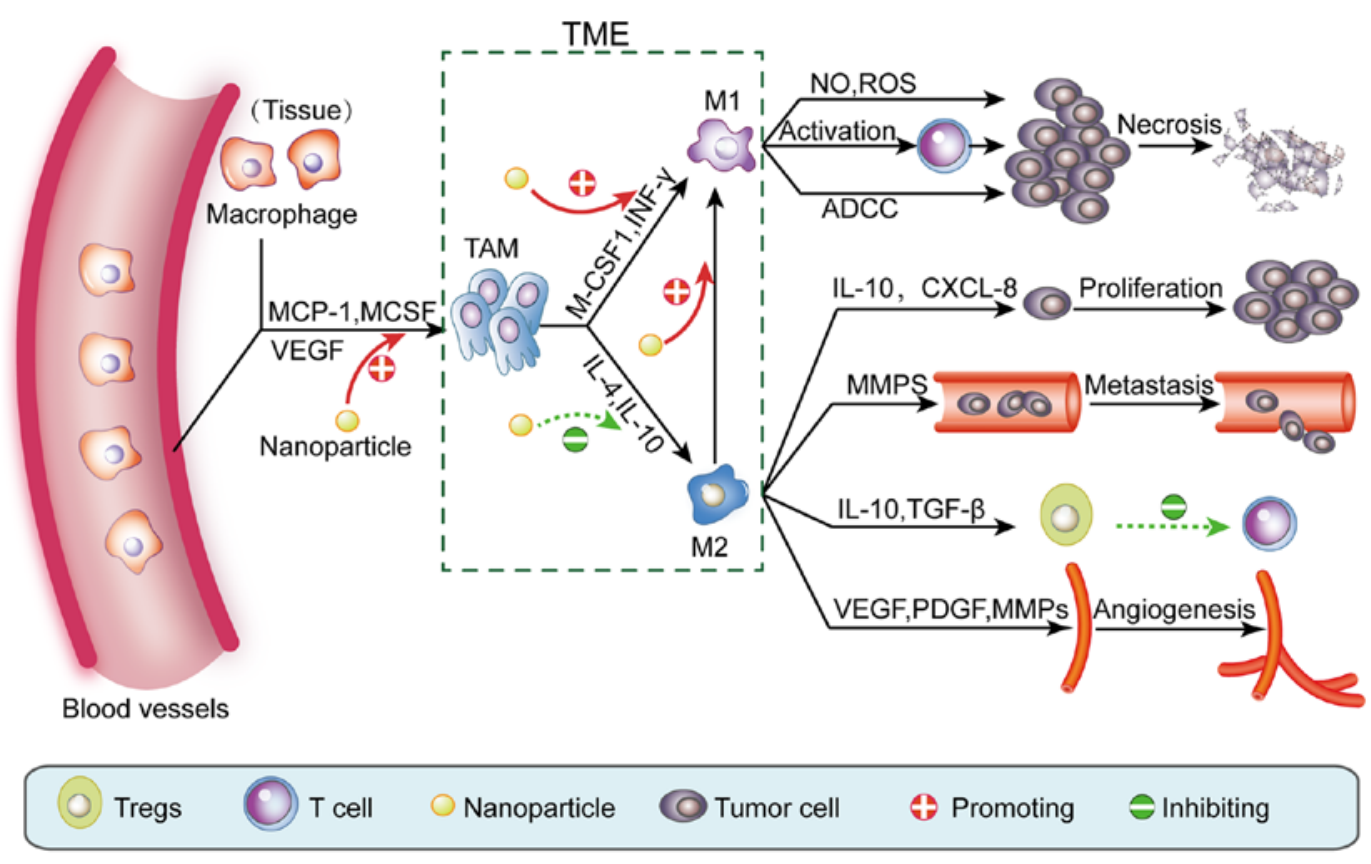

Figure 3. Nanomaterials inhibit tumors by regulating TAMs in the TME. Under the action of cytokines, blood vessels and tissue macrophages are recruited into tumor tissue and become TAMs. Similarly, under stimulation with different cytokines, TAMs are polarized to pro-inflammatory M1 macrophages or anti-inflammatory M2 macrophages. M1 macrophages have antitumor effects such as: direct elimination of tumor cells through the release of NO and ROS, ADCC and activation of T cells. In contrast, M2 macrophages can promote tumor development through i) secretion of cytokines, such as IL-10 and CXCL-8, which directly promote the growth of tumor cells; ii) secretion of MMPs that promote tumor cell infiltration and metastasis; iii) secretion of IL-10 and TGF- $\beta$, which promote Treg differentiation, thereby inhibiting T-cell activation; and iv) secretion of VEGF, PDGF and MMPs that promote tumor neovascularization. Nanomaterials can promote the recruitment of macrophages in the TME and induce TAM polarization to the M1 rather than the M2 phenotype. Furthermore, nanomaterials can also promote transformation of M2 macrophages to M1 macrophages. TAMs, tumor-associated macrophages; ADCC, antibody-dependent cell-mediated cytotoxicity; MMPs, matrix metalloproteinases; TGF- $\beta$, transforming growth factor $\beta$; VEGF, vascular endothelial growth factor; PDGF, platelet-derived growth factor; TME, tumor microenvironment.

Regulation of the tumor microenvironment (TME). TME refers to the internal environment of tumors, consisting of tumor, stromal and immune cells, microvessels and interstitium and is infiltrated by biological molecules (26). Interactions between the TME and tumor cells have an important effect on tumor growth and metastasis (27). Due to the special physical and chemical properties of nanoparticles, which can be enriched in the TME, tumor blood vessels have enhanced permeability and retention (EPR) (28). Nanoparticles in the TME can regulate the cellular and non-cellular components and then produce antitumor effects.

Targeting tumor-associated macrophages (TAMs). TAMs are macrophages that have infiltrated tumor tissue and are the most abundant immune cells in the TME. Via cytokines in the TME, macrophages differentiate into different types of TAMs, mainly divided into the M1 and M2 type (29). M1 TAMs can release a variety of pro-inflammatory cytokines, immune activators and chemokines and play an antitumor role through induction of acute inflammation, immune activation and phagocytosis via the following mechanisms: i) release of NO and ROS and direct elimination of tumor cells; ii) antibody-dependent cell-mediated cytotoxicity (ADCC); and iii) induction of a specific immune response (29). M2 type TAMs exert immunosuppressive effects through the following mechanisms: i) secretion of cytokines, such as CXCL-8 and IL-10, that promote tumor cell growth (30); ii) production of matrix metalloproteinases (MMPs) that promote tumor invasion and metastasis; iii) secretion of cytokines, such as IL-10 and TGF- $\beta$, that inhibit T-cell activation and promote the differentiation of regulatory T cells (Tregs) (31); and iv) secretion of VEGF, PDGF, and MMPs that promote neovascularization of tumor cells (32) (Fig. 3).

Zanganeh $\operatorname{etal}$ (33) found 'hidden functions' of ferumoxytol, which has been approved by the FDA for the treatment of anemia, such as the production of antitumor effects by modulating TAMs in the TME, while being non-toxic to normal cells. Experiments revealed that ferumoxytol can recruit more macrophages and induce M1 polarization. Notably, apoptotic cancer cells continue to induce M1 polarization to form an autocrine feedback loop which maintains the production of tumor cytotoxic substances, thereby killing tumor cells. In vivo, local and systemic inoculation of the nanoparticles significantly inhibited tumor cell growth as well as treated and prevented distant metastasis of the tumor. Notably, the experiments clarified that the anticancer mechanism of ferumoxytol includes the promotion of macrophage recruitment for M1 macrophage polarization, inhibiting M2 macrophages.

Targeting tumor vasculature. The rapid growth of tumors requires neovascularization to provide adequate nutrient supply. Tumor neovascularization is dependent on angiogenic factors, mainly vascular endothelial growth factor (VEGF) and platelet-derived growth factor (PDGF), which are widely overexpressed in tumors (34). Therefore, regulation of angiogenic factors can inhibit tumor growth. Some nanomaterials can modulate some of the components of the TME without the need for binding biomolecules. Numerous studies have revealed 
that gadolinium-containing fullerene nanoparticles are not toxic to normal cells, however inhibit tumor growth and metastasis $(35,36)$. Metal fullerene nanoparticles can act on multiple angiogenic factors concurrently, effectively inhibiting angiogenesis and decreasing the microvessel density in tumors (35). In addition, they significantly decreased the activity of MMPs and promoted the formation of a fiber cage, which can block signal transduction between TAMs and tumor cells (36).

Nanoparticles can kill tumor cells. Some nanomaterials, due to their particular physical and chemical properties, can kill cancer cells under certain conditions $(37,38)$. The photothermal effect of certain nanomaterials, for instance, has become a topic of great interest in cancer treatment studies $(37,39)$. Photothermal therapy refers to the use of a material with a high photothermal conversion efficiency that is injected into the body and under irradiation of an external light source (usually near infrared light), the light energy is converted into heat energy that kills cancer cells.

Guo et al (37) designed HCuSNPs for the combination of photothermal therapy and immunotherapy. After the recombination, the compound is more stable, easily remains at the tumor site and is easily taken up by DCs. Furthermore, photothermal ablation induced apoptosis of local cancer cells and some tumor antigens were released into the surrounding environment and synergized with chitosan-CPG nanocomplexes to activate antigen-specific antitumor immune responses. Similarly, Duan et al (38) revealed that Zn-pyrophosphate (ZnP) nanoparticles loaded with the photosensitizer pyrolipid (ZnP@pyro) can induce apoptosis via light irradiation, destroy the tumor vasculature and increase tumor immunogenicity.

\section{Application of nanotechnology in cancer vaccines}

Tumor cell vaccines. Tumor cell vaccines include autologous tumor whole cell vaccines and allogeneic tumor cell vaccines. Autologous tumor cell vaccines are prepared by isolating tumor cells from patients, processing them into vaccines in vitro, and then administering the vaccine to the same patient (40). Allogeneic tumor cell vaccines, which often contain two or three established human tumor cell lines, can be used to overcome many of the limitations of autologous tumor cell vaccines (41). Tumor cell vaccines can present all the tumor antigens, which induce an immune response to the tumor antigens. There are many tumor antigens on the cancer cell membrane surface and it is difficult to achieve these functions through traditional synthesis technology, although this would be an ideal vaccine (42). However, due to the low immunogenicity of tumor vaccines alone, they can only stimulate a small immune response in the body and therefore, it is essential to find a suitable adjuvant to enhance the immunogenicity of a tumor cell vaccine (43).

Fang et al (43) combined purified tumor cell membrane with PLGA nanoparticles to form cancer cell membrane-coated nanoparticles (CCNPs). Combination of monophosphoryl lipid A (MPLA) with CCNPs in a vaccine promoted the maturation of DCs, the uptake of antigen and induced immune activation. Similarly, Liu et al (44) designed a multi-adjuvant whole cell tumor vaccine (WCTV) based on PLGA nanoparticles modified with a cell penetrating peptide, which could promote transpor- tation of GM-CSF and IL-2 into tumor cells. The experimental data revealed that the compound adjuvants promoted DC recruitment, antigen presentation and $\mathrm{T}$-cell activation.

Dendritic cell vaccines. The use of DCs as the carrier in a DC cancer vaccine is recognized as one approach for tumor immunotherapy. A tumor antigen-loaded DC vaccine is considered to have enhanced potential for tumor immunotherapy (45). However, these vaccines mainly use virus or virus-like particles (VLPs) as carriers to transfer antigens into DCs. Thus, they often cause an associated immune response, producing specific neutralizing antibodies to prevent viral infection and therefore, their use is risk-bearing (46). Because of their unique physicochemical properties, nanomaterials have exhibited great potential as vaccine carriers/adjuvants to enhance the immunogenicity of antigens by promoting antigen uptake by DCs, protecting antigens from enzymatic degradation and regulating immune cells (47).

Zeng et al (48) transfected DCs with AFP1 cDNA (with a signal peptide) and AFP2 cDNA (without a signal peptide) using calcium phosphate nanoparticles and induced maturation with recombinant mouse granulocyte macrophage colony-stimulating factor (rmGM-CSF), creating a DC vaccine. The in vivo and in vitro results revealed that the $\mathrm{DC}$ vaccine induced activation and proliferation of T lymphocytes to promote secretion of the cytokine IFN- $\gamma$, which induced a highly effective and specific immune response to liver cancer. In addition, Matsuo et al (49) encapsulated OVA in biodegradable nanoparticles ( $\gamma$-PGA NPs) and delivered it to DCs in vitro. The in vivo experimental results revealed that the use of OVA $/ \gamma$-PGA NP-pulsed DCs induced TAA-specific CTLs, resulting in a strong antitumor effect.

Peptide vaccines. Cancer peptide vaccines have the advantage of direct stimulation, high specificity of the immune response, no autoimmune response or immunosuppression, as well as lack of carcinogenic risk and have wide prospective applications (50). However, cancer peptide vaccines also have shortcomings, such as weak immunogenicity, a short half-life and being prone to immune tolerance. Nanomaterials can be used as carriers in cancer peptide vaccines and as vehicles for co-delivery of antigens and immune adjuvants to target the immune system, which increases peptide vaccine immune function (51).

Zhuang et al (52) used lipid-coated zinc phosphate hybrid nanoparticles (LZnP NPs) to deliver polypeptides (TRP2180-188 and HGP10025-33) and Toll-like receptor 4 agonists. The combination of $\mathrm{H}-2 \mathrm{~K}$ (b) and $\mathrm{H}-2 \mathrm{D}$ (b)-restricted peptides provided multiple epitopes as targets of specific MHC alleles and then, the immune system was better able to monitor the tumor. The data revealed that the LZnP nanovaccine increased the secretion of cytokines and the population of $\mathrm{CD}^{+} \mathrm{T}$ cells with IFN- $\gamma$ secretion. The antitumor effect of the nanovaccine was significant in the treatment and prevention of a melanoma-mouse model compared with free antigens and a single peptide-loaded nanovaccine (52).

Genetic vaccines. Genetic vaccines, also known as nucleic acid vaccines, contain recombinant genes encoding a tumor antigen that are inserted into vectors and then introduced into host cells, which then express the exogenous antigenic proteins that 
can induce an immune response against the antigen, thereby preventing and treating the disease. Genetic vaccines are divided into DNA vaccines and RNA vaccines, however DNA vaccines are the most researched nucleic acid vaccines. DNA vaccines are promising therapeutic vaccines for tumors, but conventional vaccination with plasmids is less than ideal and has potential safety concerns (53). To this end, the use of synthetic novel non-viral vector materials for the delivery of DNA vaccines has become an intensely studied area of research $(54,55)$.

Liu et al (54) designed alginic acid-coated chitosan nanoparticles (ACNPs) as an oral delivery carrier for the legumain-DNA vaccine. The experimental data revealed that ACNPs were better than chitosan nanoparticles (CNPs) in protecting DNA from degradation in an acidic solution ( $\mathrm{pH}$ 1.5). Furthermore, the results indicated that the vaccine could avoid degradation of DNA by gastric acid and was effectively taken up and expressed by APCs and the tumor volume was significantly reduced.

Li et al (55) used four DNA strands that self-assembled into DNA nanostructures with well-defined structures and uniform sizes. Unmethylated CpG bound to the end of DNA strands by base pairing to form three-dimensional DNA tetrahedra carrying an adjuvant. Experiments demonstrated that the DNA nanostructures effectively entered macrophage-like RAW264.7 cells without transfection reagents. DNA nanostructures have high plasticity, a precise structure, stable properties, low toxicity, and resistance to nuclease degradation. Therefore, DNA nanostructures provide an unprecedented opportunity for the design of safe and effective nanovaccine carriers.

\section{Conclusions and future perspectives}

In the field of tumor immunotherapy, animal experiments have revealed that multifunctional nanomaterials combined with cancer vaccines are an effective antitumor treatment regimen. Multifunctional nanodelivery systems can simultaneously deliver tumor antigens and nanoadjuvants to draining lymph nodes and prolong antigen release time, triggering effective and lasting antitumor immune effects. The discovery of effective TAAs and their targeted delivery to APCs are keys to the development of immune responses to cancer vaccines. However, a strong antitumor immune effect may cause a potential autoimmune response, leading to severe autoimmune diseases. Therefore, it is necessary to further seek more effective tumor-specific antigens, better targeting of nanomaterials and to achieve personalized therapy for patients with new specific antigens. Simultaneously, it is difficult to eliminate the immune suppression effect of the TME and the selection of appropriate TAAs and epitopes also limits the development of cancer vaccines. At present, some nanomaterial-based cancer vaccines have entered clinical trials, such as phase 1 trials for PAN-301-1 in prostate cancer and Lipovaxin-MM in melanoma. However, most nanomaterial-based cancer vaccines remain in the experimental animal-model stage. To translate these studies from experimental animal models to clinical application, it is necessary to develop a safe and effective nano-adjuvant. Simultaneously, it is necessary to produce nanoparticles in a controllable, reproducible and scalable manner, which will be the main challenge for application of nanomaterials in cancer vaccines.
With the development of nanobiotechnology and the optimization of nanomaterials, surface modification and functionalization of nanoparticles will lead to more ideal nanomaterials and in turn to breakthroughs in the diagnosis, treatment and even prevention of human tumors. Furthermore, recent biomedical breakthroughs in tumor neoplastic antigen screening, such as tumor DNA/RNA exon sequencing and identification of new antigens, have contributed to the advancement of individualized immunotherapy for tumors. With optimized nanoadjuvants that can bind to TAAs with stronger specificity, combined with immune-related molecules, such as cytokines or immunopotentiators, nanocancer vaccines with targeted, safe and timed quantitative release can be obtained and promise to become the future focus of studies that further address immunotherapy challenges. Concurrently, the light and heat sensitivity and the magnetic properties of nanomaterials combined with immunotherapy for cancer treatment present novel therapeutic options. In the near future, nanomaterial-based cancer vaccines will be combined with surgical resection, radiotherapy, chemotherapy and other traditional treatment programs and are expected to play greater roles in clinical tumor treatment.

\section{Acknowledgements}

The present review was supported by the following grants: the NSFC 81301958 and the Science and Technology Commission Foundation of Sichuan Province LY-58.

\section{References}

1. Qasim W and Thrasher AJ: Progress and prospects for engineered T cell therapies. Br J Haematol 166: 818-829, 2014.

2. Ribas A, Butterfield LH, Glaspy JA and Economou JS: Current developments in cancer vaccines and cellular immunotherapy. J Clin Oncol 21: 2415-2432, 2003.

3. Aly HA: Cancer therapy and vaccination. J Immunol Methods 382: 1-23, 2012.

4. Lollini PL, Cavallo F, Nanni P and Forni G: Vaccines for tumour prevention. Nat Rev Cancer 6: 204-216, 2006.

5. Smith DM, Simon JK and Baker JR Jr: Applications of nanotechnology for immunology. Nat Rev Immunol 13: 592-605, 2013.

6. Pashine A, Valiante NM and Ulmer JB: Targeting the innate immune response with improved vaccine adjuvants. Nat Med 11 (Suppl): S63-S68, 2005.

7. Schlosser E, Mueller M, Fischer S, Basta S, Busch DH, Gander B and Groettrup M: TLR ligands and antigen need to be coencapsulated into the same biodegradable microsphere for the generation of potent cytotoxic T lymphocyte responses. Vaccine 26: 1626-1637, 2008.

8. De Temmerman ML, Rejman J, Demeester J, Irvine DJ, Gander B and De Smedt SC: Particulate vaccines: On the quest for optimal delivery and immune response. Drug Discov Today 16: 569-582, 2011.

9. Liu H, Moynihan KD, Zheng Y, Szeto GL, Li AV, Huang B, Van Egeren DS, Park C and Irvine DJ: Structure-based programming of lymph-node targeting in molecular vaccines. Nature 507: 519-522, 2014.

10. Palucka K and Banchereau J: Dendritic-cell-based therapeutic cancer vaccines. Immunity 39: 38-48, 2013.

11. Scheerlinck JP and Greenwood DL: Virus-sized vaccine delivery systems. Drug Discov Today 13: 882-887, 2008.

12. Goldberg MS: Immunoengineering: How nanotechnology can enhance cancer immunotherapy. Cell 161: 201-204, 2015.

13. Song YC, Cheng HY, Leng CH, Chiang SK, Lin CW, Chong P, Huang $\mathrm{MH}$ and Liu SJ: A novel emulsion-type adjuvant containing $\mathrm{CpG}$ oligodeoxynucleotides enhances $\mathrm{CD} 8^{+} \mathrm{T}-$-cellmediated anti-tumor immunity. J Control Release 173: 158-165, 2014. 
14. Fischer NO, Rasley A, Corzett M, Hwang MH, Hoeprich PD and Blanchette CD: Colocalized delivery of adjuvant and antigen using nanolipoprotein particles enhances the immune response to recombinant antigens. J Am Chem Soc 135: 2044-2047, 2013.

15. Standley SM, Mende I, Goh SL, Kwon YJ, Beaudette TT, Engleman EG and Fréchet JM: Incorporation of CpG oligonucleotide ligand into protein-loaded particle vaccines promotes antigen-specific CD8 T-cell immunity. Bioconjug Chem 18 : 77-83, 2007.

16. Fox CB, Sivananthan SJ, Duthie MS, Vergara J, Guderian JA, Moon E, Coblentz D, Reed SG and Carter D: A nanoliposome delivery system to synergistically trigger TLR4 AND TLR7. J Nanobiotechnology 12: 17, 2014

17. Zinkernagel RM, Ehl S, Aichele P, Oehen S, Kündig T and Hengartner $\mathrm{H}$ : Antigen localisation regulates immune responses in a dose- and time-dependent fashion: A geographical view of immune reactivity. Immunol Rev 156: 199-209, 1997.

18. Reddy ST, Swartz MA and Hubbell JA: Targeting dendritic cells with biomaterials: Developing the next generation of vaccines Trends Immunol 27: 573-579, 2006.

19. Cui Z, Han SJ and Huang L: Coating of mannan on LPD particles containing HPV E7 peptide significantly enhances immunity against HPV-positive tumor. Pharm Res 21: 1018-1025, 2004

20. Guo Y, Wang D, Song Q, Wu T, Zhuang X, Bao Y, Kong M, Qi Y, Tan $\mathrm{S}$ and Zhang Z: Erythrocyte membrane-enveloped polymeric nanoparticles as nanovaccine for induction of antitumor immunity against melanoma. ACS Nano 9: 6918-6933, 2015.

21. Perez-Diez A, Joncker NT, Choi K, Chan WF, Anderson CC, Lantz O and Matzinger P: CD4 cells can be more efficient at tumor rejection than CD8 cells. Blood 109: 5346-5354, 2007.

22. Delamarre L, Pack M, Chang H, Mellman I and Trombetta ES: Differential lysosomal proteolysis in antigen-presenting cells determines antigen fate. Science 307: 1630-1634, 2005.

23. Shen H, Ackerman AL, Cody V, Giodini A, Hinson ER, Cresswell P, Edelson RL, Saltzman WM and Hanlon DJ: Enhanced and prolonged cross-presentation following endosomal escape of exogenous antigens encapsulated in biodegradable nanoparticles. Immunology 117: 78-88, 2006.

24. Saluja SS, Hanlon DJ, Sharp FA, Hong E, Khalil D, Robinson E, Tigelaar R, Fahmy TM and Edelson RL: Targeting human dendritic cells via DEC-205 using PLGA nanoparticles leads to enhanced cross-presentation of a melanoma-associated antigen. Int J Nanomedicine 9: 5231-5246, 2014.

25. Yuba E, Kono Y, Harada A, Yokoyama S, Arai M, Kubo K and Kono K: The application of $\mathrm{pH}$-sensitive polymer-lipids to antigen delivery for cancer immunotherapy. Biomaterials 34: 5711-5721, 2013

26. Joyce JA: Therapeutic targeting of the tumor microenvironment. Cancer Cell 7: 513-520, 2005.

27. Mohla S: Tumor microenvironment. J Cell Biochem 101: 801-804, 2007.

28. Danhier F, Feron O and Préat V: To exploit the tumor microenvironment: Passive and active tumor targeting of nanocarriers for anti-cancer drug delivery. J Control Release 148: 135-146, 2010.

29. Martinez FO, Sica A, Mantovani A and Locati M: Macrophage activation and polarization. Front Biosci 13: 453-461, 2008.

30. Mantovani A, Sozzani S, Locati M, Allavena P and Sica A: Macrophage polarization: Tumor-associated macrophages as a paradigm for polarized M2 mononuclear phagocytes. Trends Immunol 23: 549-555, 2002.

31. Gordon S and Martinez FO: Alternative activation of macrophages: Mechanism and functions. Immunity 32: 593-604, 2010.

32. Murdoch C, Giannoudis A and Lewis CE: Mechanisms regulating the recruitment of macrophages into hypoxic areas of tumors and other ischemic tissues. Blood 104: 2224-2234, 2004.

33. Zanganeh S, Hutter G, Spitler R, Lenkov O, Mahmoudi M, Shaw A, Pajarinen JS, Nejadnik H, Goodman S, Moseley M, et al: Iron oxide nanoparticles inhibit tumour growth by inducing pro-inflammatory macrophage polarization in tumour tissues. Nat Nanotechnol 11: 986-994, 2016.

34. Connolly DT, Heuvelman DM, Nelson R, Olander JV, Eppley BL, Delfino JJ, Siegel NR, Leimgruber RM and Feder J: Tumor vascular permeability factor stimulates endothelial cell growth and angiogenesis. J Clin Invest 84: 1470-1478, 1989.

35. Meng H, Xing G, Sun B, Zhao F, Lei H, Li W, Song Y, Chen Z, Yuan $\mathrm{H}$, Wang $\mathrm{X}$, et al: Potent angiogenesis inhibition by the particulate form of fullerene derivatives. ACS Nano 4: 2773-2783, 2010.
36. Meng H, Xing G, Blanco E, Song Y, Zhao L, Sun B, Li X, Wang PC, Korotcov A, Li W, et al: Gadolinium metallofullerenol nanoparticles inhibit cancer metastasis through matrix metalloproteinase inhibition: Imprisoning instead of poisoning cancer cells. Nanomedicine (Lond) 8: 136-146, 2012

37. Guo L, Yan DD, Yang D, Li Y, Wang X, Zalewski O, Yan B and Lu W: Combinatorial photothermal and immuno cancer therapy using chitosan-coated hollow copper sulfide nanoparticles. ACS Nano 8: 5670-5681, 2014.

38. Duan X, Chan C, Guo N, Han W, Weichselbaum RR and Lin W: Photodynamic therapy mediated by nontoxic core-shell nanoparticles synergizes with immune checkpoint blockade to elicit antitumor immunity and antimetastatic effect on breast cancer. J Am Chem Soc 138: 16686-16695, 2016.

39. Chen Q, Wang C, Zhan Z, He W, Cheng Z, Li Y and Liu Z: Nearinfrared dye bound albumin with separated imaging and therapy wavelength channels for imaging-guided photothermal therapy. Biomaterials 35: 8206-8214, 2014.

40. Berger M, Kreutz FT, Horst JL, Baldi AC and Koff WJ: Phase I study with an autologous tumor cell vaccine for locally advanced or metastatic prostate cancer. J Pharm Pharm Sci 10: 144-152, 2007.

41. Guo C, Manjili MH, Subjeck JR, Sarkar D, Fisher PB and Wang XY: Therapeutic cancer vaccines: Past, present, and future. Adv Cancer Res 119: 421-475, 2013.

42. Fields RC, Shimizu K and Mulé JJ: Murine dendritic cells pulsed with whole tumor lysates mediate potent antitumor immune responses in vitro and in vivo. Proc Natl Acad Sci USA 95: 9482-9487, 1998

43. Fang RH, Hu CM, Luk BT, Gao W, Copp JA, Tai Y, O'Connor DE and Zhang L: Cancer cell membrane-coated nanoparticles for anticancer vaccination and drug delivery. Nano Lett 14: 2181-2188, 2014.

44. Liu SY, Wei W, Yue H, Ni DZ, Yue ZG, Wang S, Fu Q, Wang YQ, Ma GH and Su ZG: Nanoparticles-based multi-adjuvant whole cell tumor vaccine for cancer immunotherapy. Biomaterials 34: 8291-8300, 2013.

45. Ueda Y, Itoh T, Fuji N, Harada S, Fujiki H, Shimizu K, Shiozaki A, Iwamoto A, Shimizu T, Mazda O, et al: Successful induction of clinically competent dendritic cells from granulocyte colonystimulating factor-mobilized monocytes for cancer vaccine therapy. Cancer Immunol Immunother 56: 381-389, 2007.

46. Han JA, Kang YJ, Shin C, Ra JS, Shin HH, Hong SY, Do Y and Kang S: Ferritin protein cage nanoparticles as versatile antigen delivery nanoplatforms for dendritic cell (DC)-based vaccine development. Nanomedicine (Lond) 10: 561-569, 2014.

47. Dobrovolskaia MA and McNeil SE: Immunological properties of engineered nanomaterials. Nat Nanotechnol 2: 469-478, 2007.

48. Zeng B, Liao AJ, Lu FG, Fang WY and Wang J: Inhibition of the growth of hepatocarcinoma xenograft in Balb/c mice induced by dendritic cells immunized with AFP cDNA fragement. Zhonghua Zhong Liu Za Zhi 32: 98-102, 2010 (In Chinese).

49. Matsuo K, Ishii Y, Matsuo K, Yoshinaga T, Akashi M, Mukai Y, Yoshioka Y, Okada N and Nakagawa S: The utility of poly $(\gamma$-glutamic acid) nanoparticles as antigen delivery carriers in dendritic cell-based cancer immunotherapy. Biol Pharm Bull 33: 2003-2007, 2010.

50. Yang J, Zhang Q, Li K, Yin H and Zheng JN: Composite peptidebased vaccines for cancer immunotherapy (Review). Int J Mol Med 35: 17-23, 2015.

51. Cruz LJ, Rueda F, Cordobilla B, Simón L, Hosta L, Albericio F and Domingo JC: Targeting nanosystems to human DCs via $\mathrm{Fc}$ receptor as an effective strategy to deliver antigen for immunotherapy. Mol Pharm 8: 104-116, 2011.

52. Zhuang X, Wu T, Zhao Y, Hu X, Bao Y, Guo Y, Song Q, Li G, Tan $S$ and Zhang Z: Lipid-enveloped zinc phosphate hybrid nanoparticles for codelivery of $\mathrm{H}-2 \mathrm{~K}(\mathrm{~b})$ and $\mathrm{H}-2 \mathrm{D}(\mathrm{b})$-restricted antigenic peptides and monophosphoryl lipid A to induce antitumor immunity against melanoma. J Control Release 228: 26-37, 2016.

53. Senovilla L, Vacchelli E, Garcia P, Eggermont A, Fridman WH, Galon J, Zitvogel L, Kroemer G and Galluzzi L: Trial watch: DNA vaccines for cancer therapy. OncoImmunology 2: e23803, 2013.

54. Liu Z, Lv D, Liu S, Gong J, Wang D, Xiong M, Chen X, Xiang R and Tan X: Alginic acid-coated chitosan nanoparticles loaded with legumain DNA vaccine: Effect against breast cancer in mice. PLoS One 8: e60190, 2013.

55. Li J, Pei H, Zhu B, Liang L, Wei M, He Y, Chen N, Li D, Huang Q and Fan C: Self-assembled multivalent DNA nanostructures for noninvasive intracellular delivery of immunostimulatory $\mathrm{CpG}$ oligonucleotides. ACS Nano 5: 8783-8789, 2011. 